\title{
BENZOQUINONAS, HIDROQUINONAS E SESQUITERPENOS DE Auxemma glazioviana
}

\author{
Jose Galberto M. Costa, Otília Deusdênia L. Pessoa, Francisco José Q. Monte, Eveline A. Menezes e Telma Leda G. \\ Lemos* \\ Departamento de Química Orgânica e Inorgânica, Universidade Federal do Ceará, CP 12200, 60451-970 Fortaleza - CE \\ Raimundo Braz-Filho \\ Setor de Produtos Naturais, Centro de Ciências e Tecnologia, Universidade Estadual do Norte Fluminense, 28013-600 Campos \\ dos Goytacazes - RJ
}

Recebido em 29/3/04; aceito em 5/11/04; publicado na web em 17/2/05

\begin{abstract}
BENZOQUINONES, HYDROQUINONES AND SESQUITERPENES OF Auxemma glazioviana. Two new compounds, $\mathbf{5}$ and $\mathbf{8}$, and an epimeric mixture $\mathbf{4 a} / \mathbf{4 b}$ were isolated from hardwood of Auxemma glazioviana. Their structures and relative

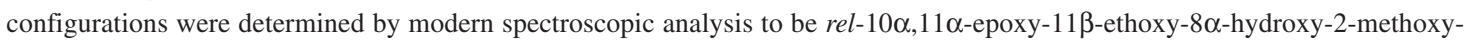
8aß-methyl-5,6,7,8,8a,9,10,10aß-octahydro-1,4-anthracenedione (or rel-2R,2aR,5R,5aS,10bS,10cS-2-ethoxy-5-hydroxy-8methoxy-5a-methyl-2a,3,4,5,5a,6,10b,10c-octahydro- $2 \mathrm{H}$-anthra[9,1-bc]furan-7,10-dione, 4a), rel-10 $\alpha, 11 \alpha$-epoxy-11 $\alpha$-ethoxy$8 \alpha$-hydroxy-2-methoxy- $8 \mathrm{a} \beta$-methyl-5,6,7,8,8a,9,10,10a $\beta$-octahydro-1,4-anthracenedione (or rel-2S,2a $R, 5 R, 5 \mathrm{a} S, 10 \mathrm{~b} S, 10 \mathrm{c} S$-2ethoxy-5-hydroxy-8-methoxy-5a-methyl-2a,3,4,5,5a,6,10b10c-octahydro-2H-anthra[9,1-bc]furan-7,10-dione, 4b), rel-10 $\alpha, 11 \alpha$ epoxy- $8 \alpha, 11$-dihydroxy-2-methoxy-8a $\beta$-methyl-5,6,7,8,8a,9,10,10a $\beta$-octahydro-1,4-anthracenedione (or rel$2 S, 2 \mathrm{a} R, 5 R, 5 \mathrm{a} S, 10 \mathrm{~b} S, 10 \mathrm{c} S$-2,5-dihydroxy-8-methoxy-5a-methyl-2a,3,4,5,5a,6,10b,10c-octahydro-2H-anthra[9, 1-bc]furan-7,10dione, 5) and rel-10,11-epoxy-8aß-methyl-1,4,5 $\alpha, 9 \alpha$-tetrahydroxy-5,6,7,8,8a,9,10,10a-octahydro-8-anthracenone (or rel$2 \mathrm{a} S, 5 \mathrm{a} S, 6 R, 10 \mathrm{~b} R, 10 \mathrm{c} 5-2 \mathrm{a}, 6,7,10$-tetrahydroxy-5a-methyl-2a,3,4,5,5a,6,10b,10c-octahydro-2H-anthra[9,1-bc]-furan-5-one, 8). In addition, known compounds were also isolated.
\end{abstract}

Keywords: Auxemma glazioviana; quinones; sesquiterpenes.

\section{INTRODUÇÃO}

Como parte de um estudo integrado entre química e farmacologia buscando descobrir novas moléculas e princípios ativos de plantas medicinais da flora nordestina, especialmente do estado do Ceará, investigou-se a constituição química das duas únicas espécies do táxon Auxemma (Boraginaceae). A. oncocalyx e A. glazioviana são conhecidas pela denominação popular de pau-branco e são bastante semelhantes, apresentando pequenas divergências tanto no habitat como em rápida análise de suas amostras de herbário. Árvores de pequeno porte, produtoras de madeira pesada e de boa qualidade, resistentes ao ataque de fungos e cupins, sendo por isto bastante empregadas na construção civil, marcenaria e carpintaria. Como consequiência da beleza de suas flores, brancas e em densas panículas terminais, são empregadas no paisagismo, na ornamentação de praças e avenidas. As cascas destes vegetais, dotadas de propriedades adstringentes, são utilizadas na medicina popular na cicatrização de cortes e feridas ${ }^{1}$. A. oncocalyx foi a primeira espécie a ser investigada, tendo sido isoladas várias quinonas e hidroquinonas terpenoídicas ${ }^{2-4}$ de esqueleto $\mathrm{C}_{16}$, estruturalmente semelhantes aos cordiacromos ${ }^{5}$, inicialmente isolados de espécies de Cordia. Dentre os compostos isolados, destaca-se a substância denominada oncocalyxona A, um pigmento de cor vinho escuro, com propriedades citotóxica ${ }^{6-8}$, genotóxica $^{9}$, anti-agregante plaquetária ${ }^{10}$, antioxidante ${ }^{11}$, analgésica e antiinflamatória ${ }^{12}$. Das cascas isolou-se quantidade significativa de alantoína ${ }^{13}$, substância com propriedades cicatrizante, antiinflamatória e regeneradora de tecidos necrosados cientificamente comprovadas ${ }^{14}$. Desta forma, acredita-se que a alantoína seja o princípio ativo das cascas deste vegetal.

*e-mail: tlemos@dqoi.ufc.br
Diante dos promissores resultados obtidos com a investigação fitoquímica de A. oncocalyx, iniciamos em paralelo a investigação fitoquímica da congênere A. glazioviana, a partir da qual vários metabólitos secundários já foram descritos na literatura, como a 1,4benzoquinona conhecida como oncocalyxona A (6), a hidroquinona denominada glaziovianol A (7), além de uma 1,4-fenantrenodiona e um diterpeno ${ }^{15}$. Além disso, registrou-se também, a composição química do óleo essencial obtido do cerne da referida espécie, juntamente com atividade larvicida contra o mosquito Aedes aegypti ${ }^{16}$.

$\mathrm{O}$ presente trabalho relata o isolamento de novos constituintes químicos do cerne do caule de A. glazioviana como, a mistura de epímeros $\mathbf{4 a}, \mathbf{4 b}$ e os compostos puros $\mathbf{5}$ e 8, os quais foram caracterizados por métodos espectroscópicos, principalmente $\mathrm{RMN}{ }^{1} \mathrm{H}$ e ${ }^{13} \mathrm{C}$, e por comparação com dados espectrais disponíveis na literatu$\mathrm{ra}^{2-4}$, bem como o isolamento dos compostos 1, 2, 3, 6 e 7 (Figura 1).<smiles>CC1=C[C@H]2C(C(C)C)CCC(O)C2CC1</smiles>

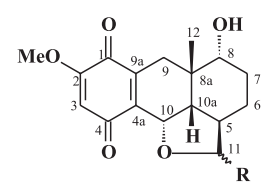

4a $\mathrm{R}=\beta-\mathrm{OEt}$ $4 \mathrm{~b} \mathrm{R}=\alpha-\mathrm{OE}$ $5 \mathrm{R}=\alpha-\mathrm{OH}$<smiles>CC(C)=CCCC(O)C1CC=CCC1</smiles><smiles>COC1=CC(=O)C2=C(CC3(C)C(=C2)C(CO)=CCC3O)C1=O</smiles>

6
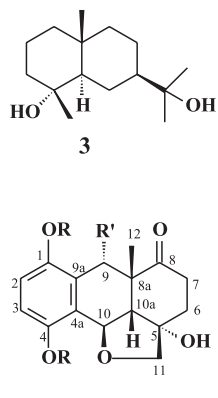

$7 \mathrm{R}=\mathrm{R}^{\prime}=\mathrm{H}$ $8 \mathrm{R}=\mathrm{H}, \mathrm{R}^{\prime}=\mathrm{OH}$ 8a $\mathrm{R}=\mathrm{Ac}, \mathrm{R}^{\prime}=\mathrm{OAc}$
Figura 1. Constituintes químicos isolados do cerne de A. glazioviana 


\section{RESULTADOS E DISCUSSÃO}

\section{Caracterização das antracenodionas 4a, 4b e 5}

O composto 4 foi obtido como um sólido amorfo amarelo, com ponto de fusão $115-117{ }^{\circ} \mathrm{C}$. O espectro de IV revelou uma banda intensa em $3400 \mathrm{~cm}^{-1}$, característica de grupo hidroxila e duas absorções em 1680 e $1670 \mathrm{~cm}^{-1}$, compatíveis com carbonilas cetônicas envolvidas em conjugação. Os espectros de $\mathrm{RMN}{ }^{1} \mathrm{H}$ e ${ }^{13} \mathrm{C}$ revelaram que o material em discussão era uma mistura epimérica. Análise detalhada dos espectros de $\mathrm{RMN}{ }^{13} \mathrm{C}$ e DEPT 135 permitiu identificar duas carbonilas, seis carbonos metínicos, dentre eles dois oximetínicos e um hemiacetálico, quatro carbonos metilênicos, um deles ligado a oxigênio, dois grupos metílicos, uma metoxila e três carbonos não-hidrogenados, permitindo assim deduzir a fórmula molecular $\mathrm{C}_{19} \mathrm{H}_{24} \mathrm{O}_{6}$, para cada epímero. Análise detalhada dos dados espectrais e comparação com valores registrados na literatu$\mathrm{ra}^{2,4}$ permitiram deduzir as estruturas dos dois compostos epiméricos, no átomo de carbono $\mathrm{C}-11$, inclusive os assinalamentos inequívocos dos sinais de hidrogênio e carbono (Tabelas 1 e 2). As principais diferenças observadas nos espectros de $\mathrm{RMN}{ }^{13} \mathrm{C}$ podem ser justificadas, com relativa facilidade, pelas modificações decorrentes dos efeitos $\alpha, \beta$ e $\gamma$ (mais importante pela influência estereoquímica, freqüentemente gerando blindagem eletrônica de proteção) revelados pela comparação dos deslocamentos químicos dos átomos de carbono localizados nas posições correspondentes [CH11: $\delta_{\mathrm{C}} 103,4(\mathbf{4 a})$ e 107,7 (4b)], [CH-5: $\delta_{\mathrm{C}} 43,6(\mathbf{4 a})$ e $\left.44,2(\mathbf{4 b})\right]$ e, $\left[\mathrm{CH}_{2}-6: \delta_{\mathrm{C}} 22,6(\mathbf{4 a})\right.$ e $24,0(\mathbf{4 b}), \Delta \delta_{\mathrm{C}}=22,6-24,0=-1,4 \mathrm{ppm}$, efeito $\gamma$ do átomo de oxigênio do grupo etoxi sobre $\mathrm{CH}_{2}-6$ em posição geometricamente favorável - cis; CH-10a: $\delta_{\mathrm{C}} 46,6$ (4a) e 48,4 (4b), $\Delta \delta_{C}=46,6-48,4=-1,8$ ppm, efeito $\gamma$ do átomo de oxigênio do grupo etoxi sobre $\mathrm{CH}-10 \mathrm{a}$ em posição geometricamente favorá-

Tabela 1. Dados de $\mathrm{RMN}{ }^{13} \mathrm{C}(125 \mathrm{MHz})$ dos compostos $\mathbf{4 a}, \mathbf{4 b}, \mathbf{5}$, 6,7 e 8

\begin{tabular}{lcccccc}
\hline $\mathrm{C}$ & $\mathbf{4 a}$ & $\mathbf{4 b}$ & $\mathbf{5}$ & $\mathbf{6}$ & $\mathbf{7}$ & $\mathbf{8}$ \\
\hline 1 & 183,3 & 182,8 & 182,6 & 180,8 & 148,0 & 149,8 \\
2 & 158,9 & 158,6 & 159,1 & 159,4 & 115,4 & 116,3 \\
3 & 108,3 & 107,9 & 107,2 & 106,0 & 113,2 & 117,0 \\
4 & 187,1 & 186,4 & 187,0 & 185,7 & 150,0 & 151,2 \\
$4 \mathrm{a}$ & 139,3 & 139,3 & 138,1 & 134,1 & 121,5 & 127,3 \\
5 & 43,7 & 44,2 & 43,3 & 135,0 & 78,8 & 80,3 \\
6 & 22,7 & 24,1 & 23,6 & 128,0 & 32,6 & 33,6 \\
7 & 31,3 & 29,9 & 22,3 & 31,6 & 35,8 & 38,1 \\
8 & 77,8 & 76,4 & 76,8 & 69,7 & 215,1 & 214,3 \\
$8 \mathrm{a}$ & 37,9 & 37,5 & 37,2 & 38,9 & 41,6 & 47,9 \\
9 & 24,2 & 24,3 & 30,1 & 28,8 & 33,1 & 67,5 \\
$9 \mathrm{a}$ & 138,5 & 137,6 & 138,6 & 132,7 & 123,3 & 122,9 \\
10 & 71,2 & 70,0 & 70,9 & 146,4 & 73,4 & 75,0 \\
$10 \mathrm{a}$ & 46,6 & 48,5 & 45,7 & 135,1 & 53,1 & 48,8 \\
11 & 103,5 & 107,7 & 97,5 & 61,2 & 82,3 & 87,9 \\
12 & 24,3 & 22,4 & 22,9 & 20,9 & 19,5 & 20,0 \\
13 & 63,6 & 65,4 & - & - & - & - \\
14 & 15,5 & 14,3 & - & - & - & - \\
$\mathrm{MeO}-2$ & 56,6 & 56,2 & 55,5 & 56,3 & - & - \\
\hline
\end{tabular}

Tabela 2. Dados de $\mathrm{RMN}{ }^{1} \mathrm{H}(500 \mathrm{MHz})$ dos compostos 4a, 4b, 5, 6, 7 e 8

\begin{tabular}{|c|c|c|c|c|c|c|}
\hline $\mathrm{H}$ & $4 a$ & $4 b$ & 5 & 6 & 7 & 8 \\
\hline 1 & - & - & - & - & - & - \\
\hline 2 & & & - & - & $6,50(\mathrm{~d}, 8,2)$ & 7,08 (d, 8,5) \\
\hline 3 & $5,94(s)$ & $5,94(s)$ & $5,90(\mathrm{~s})$ & $6,00(\mathrm{~s})$ & $6,49(\mathrm{~d}, 8,2)$ & $7,13(\mathrm{~d}, 8,5)$ \\
\hline 4 & - & - & - & - & - & - \\
\hline $4 a$ & - & - & - & - & - & - \\
\hline 5 & $1,62(\mathrm{~m})$ & $1,63(\mathrm{~m})$ & $1.75(\mathrm{~m})$ & - & - & - \\
\hline \multirow[t]{2}{*}{6} & $1,79(\mathrm{~m})$ & $1,97(\mathrm{~m})$ & $1,80-1,41$ & $6,03(\mathrm{~d}, 3,9)$ & $2,30(\mathrm{t}, 14,2)$ & $2,49(\mathrm{~m})$ \\
\hline & $1,50(\mathrm{~m})$ & $1,29(\mathrm{~m})$ & & & $2,07(\mathrm{~m})$ & \\
\hline \multirow[t]{2}{*}{7} & $1,94(\mathrm{~m})$ & $1,95(\mathrm{~m})$ & $1,97-1,63$ & $2,50(\mathrm{dl}, 17,2)$ & $2,63(\mathrm{~m})$ & $3,12(\mathrm{~m})$ \\
\hline & $1,57(\mathrm{~m})$ & $1,61(\mathrm{~m})$ & & $2,30(\mathrm{dd}, 17,2 ; 3,9)$ & $2,33(\mathrm{~m})$ & $2,58(\mathrm{dl}, 16,8)$ \\
\hline 8 & $3,55(\mathrm{dd}, 10,7 ; 4,7)$ & $3,51(\mathrm{~m})$ & $3,40(\mathrm{dd}, 4,7 ; 11,3)$ & $3,56(\mathrm{sl})$ & - & - \\
\hline $8 \mathrm{a}$ & - & - & & - & - & - \\
\hline \multirow[t]{2}{*}{9} & $2,48(\mathrm{~d})$ & $2,50(\mathrm{~d}, 19,9)$ & $2,44-2,16$ & $2,94(\mathrm{~d}, 18,4)$ & $2,97(\mathrm{~d}, 16,5)$ & $6,24(s)$ \\
\hline & $2,35(\mathrm{~d})$ & $2,17(\mathrm{dd}, 19,9 ; 2,3)$ & & $2,35(\mathrm{~d}, 18,4)$ & $2,04(\mathrm{~d}, 16.5)$ & \\
\hline $9 \mathrm{a}$ & - & - & & - & - & - \\
\hline 10 & $5,10(\mathrm{~d}, 7,7)$ & $4,97(\mathrm{dl}, 7,2)$ & $5,00(\mathrm{~d}, 7,5)$ & - & $5,04(\mathrm{~d}, 3,3)$ & $6,16(d, 5,3)$ \\
\hline $10 \mathrm{a}$ & $2,19(\mathrm{dd})$ & $1,89(\mathrm{dd}, 12,9 ; 7,2)$ & $2,10(\mathrm{dd}, 7,5 ; 12,9)$ & - & $2,13(\mathrm{~d}, 3,3)$ & $4,01(\mathrm{~d}, 5,3)$ \\
\hline \multirow[t]{2}{*}{11} & $4,87(\mathrm{~d}, 4,2)$ & $4,79(\mathrm{~d}, 7,3)$ & $5,10(\mathrm{~d}, 4,3)$ & $4,17(\mathrm{sl})$ & $3,85(\mathrm{sl})$ & $4,56(\mathrm{~d}, 9,1)$ \\
\hline & & & & & & $4,27(\mathrm{~d}, 9,1)$ \\
\hline 12 & $1,05(\mathrm{~s})$ & $1,03(\mathrm{~s})$ & $1,00(\mathrm{~s})$ & $0,73(\mathrm{~s})$ & $0,92(s)$ & $1,13(\mathrm{~s})$ \\
\hline 13 & $3,85(\mathrm{~m}) 3,46(\mathrm{~m})$ & $3,80(\mathrm{~m}) 3,49(\mathrm{~m})$ & - & - & - & - \\
\hline 14 & $1,15(\mathrm{t}, 7,0)$ & $1,15(\mathrm{t}, 7,2)$ & - & - & - & - \\
\hline $\mathrm{MeO}-2$ & $3,80(\mathrm{~s})$ & $3,81(\mathrm{~s})$ & $3,80(\mathrm{~s})$ & $3,77(\mathrm{~s})$ & - & - \\
\hline $\mathrm{HO}-1$ & - & - & - & - & $8,61(\mathrm{~s})$ & $10,90(\mathrm{sl})$ \\
\hline $\mathrm{HO}-4$ & - & - & - & - & $8,45(\mathrm{~s})$ & $10,00(\mathrm{sl})$ \\
\hline HO-5 & - & - & - & - & $5,23(\mathrm{~s})$ & - \\
\hline $\mathrm{HO}-8$ & $4,80(\mathrm{~d}, 4,5)$ & $4,80(\mathrm{~d}, 4,5)$ & $4,80(\mathrm{~d}, 4,5)$ & $4,93(\mathrm{~d}, 4,5)$ & - & - \\
\hline $\mathrm{HO}-9$ & - & - & - & - & - & $6,98(s)$ \\
\hline HO-11 & - & - & $4,80(\mathrm{~d}, 5,2)$ & $4,87(\mathrm{t}, 5,2)$ & - & - \\
\hline
\end{tabular}

Deslocamentos químicos $(\delta)$ em ppm e constantes de acoplamento $(J)$ em $\mathrm{Hz}$ 
vel - cis]. A análise comparativa dos dados espectrais dos dois epímeros (4a) e (4b), principalmente de RMN (Tabelas 1 e 2), foi utilizada para a elucidação estrutural de cada um destes produtos, os quais foram determinados com base em experimentos de RMN 1D e 2D. Assim, as estruturas dos compostos em discussão foram deduzidas como rel-10 $\alpha, 11 \alpha$-epoxi-11 $\beta$-etoxi- $8 \alpha$-hidroxi-2metoxi-8a $\beta$-metil-5,6,7,8,8a,9,10,10a $\beta$-octaidro-1,4-antracenodiona (ou rel-2R,2a $R, 5 R, 5 \mathrm{a} S, 10 \mathrm{~b} S, 10 \mathrm{c} S$-2-etoxi-5-hidroxi-8metoxi-5a-metil-2a,3,4,5,5a,6,10b,10c-octaidro-2H-anthra[9,1bc]furan-7,10-diona, 4a), e rel-10 $\alpha, 11 \alpha$-epoxi-11 $\alpha$-etoxi- $8 \alpha$ hidroxi-2-metoxi-8a $\beta$-metil-5,6,7,8,8a,9,10,10a $\beta$-octaidro-1,4antracenodiona (ou rel-2S,2aR,5R,5aS,10bS,10cS-2-etoxi-5-hidroxi8-metoxi-5a-metil-2a,3,4,5,5a,6,10b10c-octaidro-2H-anthra[9,1bc]furan-7,10-diona,4b), tendo sido designadas de $11 \alpha$-oncocalyxona $\mathrm{C}$ (previamente isolada de $A$. oncocaly $x^{3}$ ) e $11 \beta$-oncocalyxona $\mathrm{C}$, respectivamente. Acreditamos que estas substâncias tenham sido produzidas durante o processo de extração com etanol conforme ilustrado no Esquema 1.
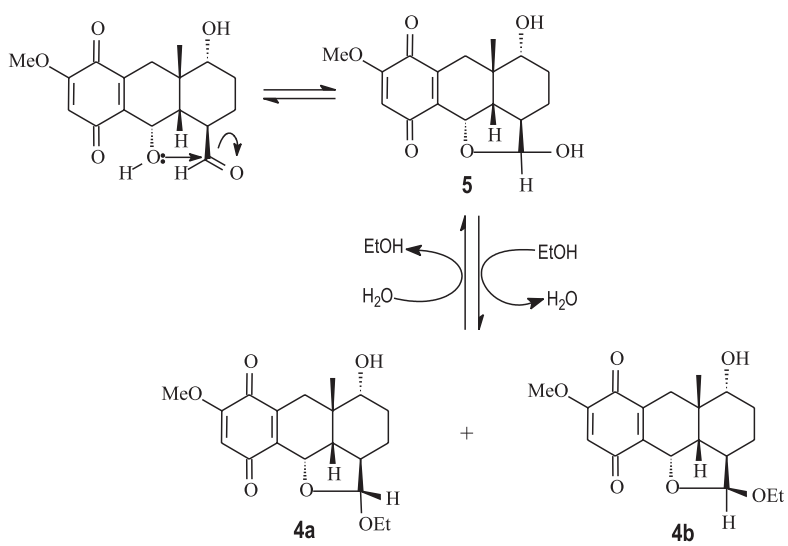

Esquema 1. Proposta para formação dos hemiacetais $\mathbf{4 a}$ e $\mathbf{4 b}$ produzidos provavelmente durante o processo de extração, usando EtOH como solvente

O composto 5 foi isolado como um sólido amorfo branco, com ponto de fusão $>290{ }^{\circ} \mathrm{C}$. Seu espectro de IV revelou a presença de grupos hidroxila e carbonila através das absorções em 3435 e $1698 \mathrm{~cm}^{-1}$, respectivamente. A fórmula molecular $\mathrm{C}_{17} \mathrm{H}_{20} \mathrm{O}_{6}$ foi deduzida a partir dos espectros de RMN ${ }^{13} \mathrm{C}$ e DEPT 135. Os sinais de $\mathrm{RMN}{ }^{1} \mathrm{H}$ e ${ }^{13} \mathrm{C}$ apresentaram-se muito próximos daqueles dos compostos $4 \mathbf{a}$ e $\mathbf{4 b}$, revelando analogia entre estes compostos (Tabelas 1 e 2). A principal diferença foi a ausência dos sinais correspondentes aos átomos de hidrogênio e carbono relativos ao grupo etoxila, presentes naquelas substâncias. Entretanto, os deslocamentos químicos em $\delta_{\mathrm{H}} / \delta_{\mathrm{C}} 5,17 / 97,5$ justificam a presença de uma hidroxila em C-11. Com base nos dados espectrais, incluindo ${ }^{1} \mathrm{H}$, ${ }^{1} \mathrm{H}-\mathrm{COSY}, \mathrm{HMQC}, \mathrm{HMBC}$ (Figura 2), NOESY e comparação com dados descritos na literatura para oncocalyxona A (6) e compostos análogos ${ }^{2,4}$, a estrutura e configuração relativa do composto 5 foi estabelecida como rel-10 $\alpha, 11 \alpha$-epoxi- $8 \alpha, 11 \alpha$-diidroxi-2-metoxi-

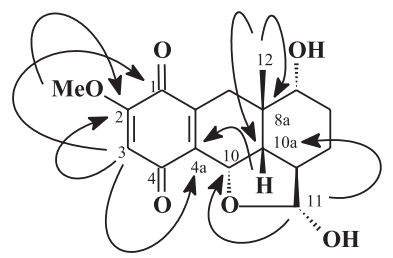

Figura 2. Correlações relevantes observadas no espectro $\mathrm{HMBC}$ de 5 8a $\beta$-metil-5,6,7,8,8a,9,10,10a $\beta$-octaidro-1,4-antracenodiona, (ou rel-2S,2a $R, 5 R, 5 \mathrm{a} S, 10 \mathrm{~b} S, 10 \mathrm{c} S$-2,5-diidroxi-8-metoxi-5a-metil2a,3,4,5,5a,6,10b,10c-octaidro-2H-anthra[9,1-bc]furan-7,10-diona,) um novo produto natural, denominado de oncocalyxona D.

\section{Caracterização da hidroquinona 8}

O composto 8 foi isolado como um sólido amorfo branco, com ponto de fusão acima de $300{ }^{\circ} \mathrm{C}$. Seu espectro de IV apresentou uma absorção intensa centrada em $3450 \mathrm{~cm}^{-1}$ característica de grupo hidroxila, uma absorção em $1696 \mathrm{~cm}^{-1}$ de carbonila conjugada, além das absorções em 1640 e $1624 \mathrm{~cm}^{-1}$ de dupla ligação. A fórmula molecular $\mathrm{C}_{16} \mathrm{H}_{18} \mathrm{O}_{6}$ foi determinada com base na análise dos espectros de RMN ${ }^{13} \mathrm{C}$ (Tabela 1), DEPT 135 e RMN ${ }^{1} \mathrm{H}$ (Tabela 2), incluindo dados espectrais como RMN ${ }^{1} \mathrm{H}$ e EM do derivado tri-acetilado 8a. O espectro de massa de $\mathbf{8}$ não revelou o pico correspondente ao íon molecular, entretanto mostrou o pico em $\mathrm{m} / z, 288$, compatível com a perda de uma molécula de $\mathrm{H}_{2} \mathrm{O}$. O espectro de $\mathrm{RMN}{ }^{1} \mathrm{H}$ revelou dois sinais em $\delta$ 7,08 (H-2) e 7,13 (H-3), ambos aparecendo como dubleto $(J=8,5 \mathrm{~Hz})$, característicos de acoplamento orto; sinais em $\delta 6,24$ (s H-9) e 6,16 (d, $J=5,3 \mathrm{~Hz}, \mathrm{H}-10$ ), compatíveis com hidrogênios ligados a carbono carbinólico; e sinais em $\delta$ 4,56 e 4,27, ambos com $J=9,1 \mathrm{~Hz}$, portanto, revelando acoplamento geminal. Observaram-se também outros sinais referentes a hidrogênios metilênicos e metínico, além dos sinais em $\delta$ 10,90 (sl), 10,00 (sl)e 6,98 (s), referentes a hidrogênios de grupo hidroxila. Os espectros de RMN ${ }^{13} \mathrm{C}$ e DEPT 135 revelaram a presença de uma carbonila $(\delta$ 214,3), cinco carbonos metínicos, dentre os quais dois com hibridação $\mathrm{sp}^{2}$ e dois carbinólicos, três carbonos metilênicos, sendo um oxigenado, dois carbonos não-hidrogenados e uma metila (Tabela 1). Estes dados, quando comparados com aqueles registrados para o metabólito secundário denominado glaziovianol (7), mostraram-se semelhantes, exceto pela presença adicional de uma hidroxila, a qual foi localizada em C-9, com base nas correlações dos átomos de hidrogênio H-10a e 3H-12 com aquele carbono, observadas no espectro HMBC (Figura 3). A estereoquímica relativa para este composto foi estabelecida com base nas correlações observadas no experimento NOESY (Figura 4). Deste modo, a estrutura deste novo produto natural, denominado glaziovianol B (8), foi definida como rel-10,11-

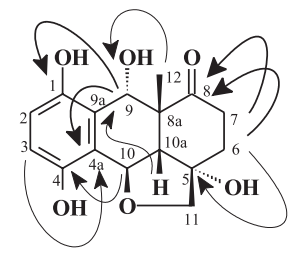

Figura 3. Correlações relevantes observadas no espectro $H M B C$ de 8

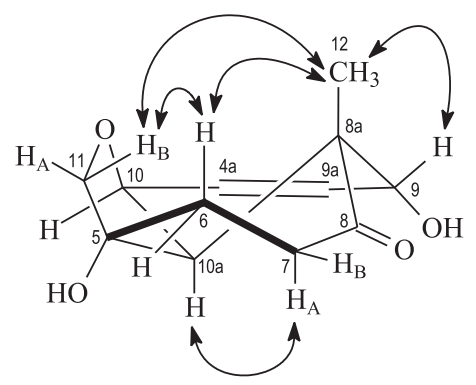

Figura 4. Configuração relativa de glaziovianol $B(\mathbf{8})$, deduzida com base no espectro NOESY 
epoxi-8aß-metil-1,4,5 $\alpha, 9 \alpha$-tetraidroxi-5,6,7,8,8a,9,10,10a-octaidro8-antracenona (ou rel-2aS, $5 \mathrm{aS}, 6 R, 10 \mathrm{~b} R, 10 \mathrm{c} 5-2 \mathrm{a}, 6,7,10$-tetraidroxi5a-metill-2a,3,4,5,5a,6,10b,10c-octaidro-2H-anthra[9,1-bc]-furan-5ona,). Os dados espectrais de $\mathrm{RMN}{ }^{1} \mathrm{H}$ (Tabela 2), inclusive o espectro de massa do derivado acetilado (8a), o qual mostra o pico correspondente ao íon molecular em $\mathrm{m} / \mathrm{z}$ 432, justificam a introdução de três grupos acilas nas posições 1,4 e 9 confirmando, desta forma, a estrutura proposta.

\section{Caracterização dos sesquiterpenos 1, 2 e 3}

Os sesquiterpenos isolados do cerne de A. glazioviana foram caracterizados por métodos espectroscópicos, principalmente RMN, e por comparação destes dados com valores descritos na literatura. Assim, os sesquiterpenos foram inequivocamente caracterizados como sendo $\alpha$-cadinol $(\mathbf{1})^{17}, \alpha$-bisabolol $(\mathbf{2})^{18}$ e proximadiol $(\mathbf{3})^{19}$. É importante ressaltar que $\alpha$-cadinol e $\alpha$-bisabolol constituem os componentes químicos majoritários dos óleos essenciais, obtidos do cerne de A. glazioviana ${ }^{16}$.

\section{PARTE EXPERIMENTAL}

\section{Procedimentos experimentais gerais}

Os pontos de fusão foram determinados em equipamento da Micro Química, modelo MQAFP 301. Os espectros de absorção no IV foram obtidos em espectrômetro Perkin-Elmer, modelo 1000FT, utilizando-se pastilhas de $\mathrm{KBr}$. Os espectros de $\mathrm{RMN}{ }^{1} \mathrm{H} \mathrm{e}{ }^{13} \mathrm{C}$, uni- e bidimensionais, foram obtidos em espectrômetros Bruker, Modelos DPX-300 (300 MHz para ${ }^{1} \mathrm{H}$ e $75 \mathrm{MHz}$ para ${ }^{13} \mathrm{C}$ ) e AVANCE DRX-500 (500 MHz para ${ }^{1} \mathrm{H}$ e $125 \mathrm{MHz}$ para ${ }^{13} \mathrm{C}$ ). Os espectros de massa das substâncias isoladas foram obtidos por impacto eletrônico a $70 \mathrm{eV}$, em espectrômetro de baixa resolução SHIMADZU, modelo QP 5000. Nas cromatografias de adsorção em coluna (CC) foi utilizado gel de sílica $(0,063-0,200 \mathrm{~mm}, 70-$ 230 mesh, VETEC), enquanto para as cromatografias de camada delgada analítica (CCDA) foram utilizadas cromatoplacas de gel de sílica, $1.05735,60 \mathrm{~A}^{\circ}$, com indicador de fluorescência na faixa de 254 ๆm (MERCK). As substâncias foram reveladas sob luz ultravioleta (254 e 366 ๆm), e/ou pela aspersão com solução de vanilina/ácido perclórico/EtOH, seguido de aquecimento, ou ainda pela exposição a vapores de iodo.

\section{Material vegetal}

Auxemma glazioviana foi coletada no município de Cristais, estado do Ceará, e identificada pelo Prof. A. G. Fernandes. A exsicata (\# 18.639) correspondente à coleta da planta encontra-se catalogada no Herbário Prisco Bezerra (EAC), do Depto. de Biologia da UFC.

\section{Extração e isolamento}

Inicialmente, o caule de A. glazioviana recém coletado, foi dividido em alburno e cerne. $\mathrm{O}$ cerne $(1,1 \mathrm{~kg})$ foi seco à temperatura ambiente, triturado e extraído exaustivamente com EtOH $(2 \times 6$ L) a frio, fornecendo $81 \mathrm{~g}$ de extrato bruto, após evaporação do solvente sob pressão reduzida. $\mathrm{O}$ extrato obtido foi fracionado sobre $150 \mathrm{~g}$ de gel de sílica, utilizando hexano, $\mathrm{CHCl}_{3}, \mathrm{AcOEt}$ e $\mathrm{MeOH}$ como eluentes. As frações resultantes foram concentradas sob pressão reduzida fornecendo os seguintes pesos: fração hexano $(3,2 \mathrm{~g})$, fração $\mathrm{CHCl}_{3}(6,0 \mathrm{~g})$, fração AcOEt $(50,8 \mathrm{~g})$ e fração $\mathrm{MeOH}(14,6 \mathrm{~g})$. A fração hexano (3,2 $\mathrm{g}$ ) foi cromatografada sobre $20 \mathrm{~g}$ de gel de sílica e eluida com hexano, hexano-AcOEt (9:1, 8:2, 7:3, 6:4, 8:2) e AcOEt. As frações iniciais, obtidas por eluição com hexano, proporcionaram o isolamento de $\mathbf{1}(220 \mathrm{mg})$, enquanto das frações obtidas por eluição com hexano-AcOEt (9:1) isolou-se o composto $2(55 \mathrm{mg})$. A fração $\mathrm{CHCl}_{3}(6,0 \mathrm{~g})$ foi submetida à cromatografia em coluna de gel de sílica (40 g) e eluida com hexano-AcOEt (10:0, 8:2, 6:4, 4:6, 8:2, 0:10), seguido de $\mathrm{MeOH}$. Nas frações obtidas por eluição com hexano-AcOEt (6:4) houve a formação de um precipitado que, após filtração e recristalização em acetona, forneceu o composto 3 (9 mg). As frações obtidas com hexano-AcOEt (4:6) foram reunidas resultando em 2,0 g de material, o qual foi recromatografado eluindo-se com hexano-AcOEt em misturas de polaridade crescente, obtendo-se os compostos 4 (22 mg) e 5 (19 mg), por eluição com hexano-AcOEt (1:1). Nas frações obtidas com AcOEt, formou-se um precipitado que, após filtração, resultou no isolamento de 6 (920 mg). A fração AcOEt (50 g) foi cromatografada em $80 \mathrm{~g}$ de gel de sílica, utilizando hexano, AcOEt e $\mathrm{MeOH}$, puros ou em misturas binárias de polaridade crescente. As frações obtidas por eluição com hexano-AcOEt (6:4), após analise em CCD, foram reunidas e o material resultante $(590 \mathrm{mg})$, recromatografado, resultando por eluição com hexano-AcOEt (4:6) no isolamento de 7 (134 mg). Nas frações obtidas por eluição com hexano-AcOEt (2:8), houve a formação de um precipitado. Estas frações foram reunidas e o resíduo recromatografado sobre gel de sílica, eluindo-se com misturas de hexano-AcOEt, resultando por eluição com hexano-AcOEt (3:7) no isolamento de 8 (30 mg).

$\alpha$-Cadinol (1). Aspecto físico: cristais incolores em forma de agulhas, p.f.: $74,3-75,9^{\circ} \mathrm{C}$, IV (KBr): $v_{\max } 3346,2960,2900,1450$, $1130 \mathrm{~cm}^{-1}$; EM m/z (int. rel.): 222 (8), 204 (45), 161 (55), 121 (54), 105 (62), 95 (85), 43 (100), 41(90); $\mathrm{RMN}^{13} \mathrm{C}\left(125 \mathrm{MHz}, \mathrm{CDCl}_{3}\right): \delta_{c}$ 50,3 (C-1), 23,0 (C-2), 31,3 (C-3), 134,8 (C-4), 122,7 (C-5), 40,2 (C-6), 47,0 (C-7), 22,3 (C-8), 42,5 (C-9), 72,8 (C-10), 26,3 (C-11), 21,9 (C-14), 15,5 (C-13), 21,1 (C-14), 24,2 (C-15).

$\alpha$-Bisabolol (2). Aspecto físico: óleo incolor, IV (filme): $v_{\max }$ 3396, 1644, 1256, $1120 \mathrm{~cm}^{-1} \mathrm{EM} \mathrm{m/z}$ (int. rel.): 204 (6), 119 (62) 109 (72), 93 (47), 69 (78), 43(100); RMN ${ }^{13} \mathrm{C}\left(125 \mathrm{MHz}, \mathrm{CDCl}_{3}\right)$ :

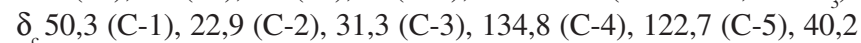
(C-6), 47,0 (C-7), 22,3 (C-8), 42,5 (C-9), 72,8 (C-10), 26,3 (C-11), 21,9 (C-12), 15,5 (C-13), 21,1 (C-14), 24,2 (C-15).

Proximadiol (3). Aspecto físico: óleo incolor, IV (filme): $v_{\max }$ 3380, 2870, 1467, $1183 \mathrm{~cm}^{-1} ;{ }^{13} \mathrm{C}\left(125 \mathrm{MHz}, \mathrm{CDCl}_{3}\right): \delta_{\mathrm{c}} 41,1(\mathrm{C}-1)$, 20,3 (C-2), 43,5 (C-3), 72,7 (C-4), 54,8 (C-5), 21,6 (C-6), 50,0 (C7), 22,8 (C-8), 44,7 (C-9), 34,6 (C-10), 73,3 (C-11), 27,6 (C-12), 26,8 (C-13), 18,9 (C-14), 22,6 (C-15).

$11 \alpha$-Oncocalyxona C (4a). Aspecto físico: sólido amorfo amarelado, p.f.: $115-117{ }^{\circ} \mathrm{C}$, IV $(\mathrm{KBr}): v_{\max } 3450,1680,1660,1450$, 1200, $1110 \mathrm{~cm}^{-1}$; EM m/z (int. rel.): 348, ausente, 303 (8) 274 (65), 256 (17), 241 (27); RMN ${ }^{13} \mathrm{C}$ (125 MHz) e ${ }^{1} \mathrm{H}$ (500 MHz), Tabelas 1 e 2 , respectivamente.

11ß-Oncocalyxona C (4b). Aspecto físico: sólido amorfo amarelado, p.f.: $111-114{ }^{\circ} \mathrm{C}$, IV $(\mathrm{KBr}): v_{\max } 3400,1676,1648,1608$, 1455, 1224, $1075 \mathrm{~cm}^{-1}$; EM m/z (int. rel.): 348, ausente, 303 (12) 274 (65), 256 (24), 241 (35); RMN ${ }^{1} \mathrm{H}(500 \mathrm{MHz})$ e ${ }^{13} \mathrm{C}(125 \mathrm{MHz})$, Tabelas 1 e 2, respectivamente.

Oncocalyxona D (5). Aspecto físico: sólido amorfo branco, p.f.: 269-271 ${ }^{\circ} \mathrm{C}, \mathrm{IV}(\mathrm{KBr}): v_{\max } 1698,1609,1483,1257,1068 \mathrm{~cm}^{-1}$; $\mathrm{RMN}{ }^{1} \mathrm{H}(500 \mathrm{MHz})$ e ${ }^{13} \mathrm{C}(125 \mathrm{MHz})$, Tabelas 1 e 2, respectivamente.

Oncocalyxona A (6). Aspecto físico: sólido amorfo de coloração vinho, p.f.: 209-211 ${ }^{\circ} \mathrm{C}$; IV (KBr): $v_{\max } 3430,3380,1645,1625$, 1570, 1485, 970, $845 \mathrm{~cm}^{-1}$; EM m/z (int. rel.): 302 (50), 284 (30), 255 (100), 240 (41), 203 (12), 171 (22), 128 (31), 115 (31); RMN ${ }^{1} \mathrm{H}(500 \mathrm{MHz})$ e ${ }^{13} \mathrm{C}(125 \mathrm{MHz})$, Tabelas 1 e 2, respectivamente. 
Glaziovianol A (7). Aspecto físico: sólido amorfo branco, p.f.: acima $290{ }^{\circ} \mathrm{C}$; IV (KBr): $v_{\max } 3436,3225,1698,1618,1483,1312$, $1207 \mathrm{~cm}^{-1} ; \mathrm{EM} \mathrm{m} / z$ (int. rel.): 290 (75), 259 (18), 175 (100), 160 (15); RMN ${ }^{1} \mathrm{H}(500 \mathrm{MHz})$ e ${ }^{13} \mathrm{C}(125 \mathrm{MHz})$, Tabelas 1 e 2, respectivamente.

Glaziovianol B (8). Aspecto físico: sólido amorfo branco, p.f.: acima de $300{ }^{\circ} \mathrm{C}$; IV (KBr): $v_{\max } 3450,3400,1696,1624,1479$, 1262, $1067 \mathrm{~cm}^{-1}$; EM m/z (int. rel.): 306 (ausente), 288 (17), 259 (15), 187 (17), 175 (100), 160 (21), 131 (19), 115 (24); RMN ${ }^{1} \mathrm{H}$ $(500 \mathrm{MHz})$ e ${ }^{13} \mathrm{C}(125 \mathrm{MHz})$, Tabelas 1 e 2, respectivamente.

Glaziovianol B 1,4,9-tri-acetilado (8a). Aspecto físico: resinoso, IV (KBr): $v_{\max } 3482,2921,2948,1751,1653,1627,1229,1189$ $\mathrm{cm}^{-1}$; EM m/z (int. rel.): 432 (0,6), 390 (4), 372 ( 2), 354 (5), 312 (4), 270 (31), 252 (21), 242 (9), 43 (100); RMN ${ }^{1} \mathrm{H}(500 \mathrm{MHz})$ : $7,13$ (d, $J=8,7 \mathrm{~Hz}, \mathrm{H}-2), 7,20$ (d, $J=8,7 \mathrm{~Hz}, \mathrm{H}-3), 2,35$ (m, 2H-6), 3,21 (d, $J=9,1 \mathrm{~Hz}, \mathrm{H}-7 \mathrm{ax}$ ), 2,51 (m, H-7eq), 6,56 (s, H-9), 5,13 (d, $J=5,1 \mathrm{~Hz}, \mathrm{H}-10), 3,22$ (d, $J=5,1 \mathrm{~Hz}, \mathrm{H}-10 \mathrm{a}), 4,42$ (d, $J=11,4 \mathrm{~Hz}$, $\mathrm{H}-11$ ), 4,29 (d, $J=11,4 \mathrm{~Hz}, \mathrm{H}-11), 1,09$ (s, H-12), 2,33 (s, $\mathrm{CH}_{3}$ $\mathrm{CO}), 2,19$ (s, $\left.\mathrm{CH}_{3}-\mathrm{CO}\right), 1,92\left(\mathrm{~s}, \mathrm{CH}_{3}-\mathrm{CO}\right)$.

\section{AGRADECIMENTOS}

Os autores agradecem às instituições de fomento à pesquisa CNPq, CAPES, FUNCAP, PRONEX e FAPERJ pelo apoio financeiro e ao Prof. E. R. Silveira (CENAUREMN-UFC) pela obtenção dos espectros de RMN.

\section{REFERÊNCIAS}

1. Braga. R.; Plantas do Nordeste Especialmente do Ceará, Coleção Mossoroense, $3^{\text {a }}$ ed., RN, 1976, p. 395.
2. Pessoa, O. D. L.; Lemos, T. L. G.; Silveira, E. R.; Braz-Filho, R.; Nat. Prod. Lett. 1993, 2, 145.

3. Pessoa, O. D. L.; Lemos, T. L. G.; Carvalho, M. G.; Braz-Filho, R.; Phytochemistry 1995, 40, 1777.

4. Marques, W. B.; Santos, H. S.; Pessoa, O. D. L.; Braz-Filho, R.; Lemos, T. L. G.; Phytochemistry 2000, 55, 793.

5. Moir, M.; Thomson, R. H.; J. Chem. Soc., Perkin Trans. 1 1973, 1352.

6. Pessoa, C.; Silveira, E. R.; Lemos, T. L. G.; Wetmore, L. A.; Moraes, M. O.; Leyva, A.; Phytotherapy Res. 2000, 14, 187.

7. Leyva, A.; Pessoa, C.; Boogaerdt, F.; Sokaroski, R.; Lemos, T. L. G.; Wetmore, L. A.; Huruta, R. R.; Moraes, M. O.; Anticancer Res. 2000, 20, 102.

8. Costa-Lotufo, L. V.; Ferreira, M. A. D.; Lemos, T. L. G.; Pessoa, O. D. L.; Viana, G. S. B.; Cunha, G. M. A.; Braz. J. Med. Biol. Res. 2002, 35, 927.

9. Pessoa, C.; Vieira, F. M. A. C.; Lemos, T. L. G.; Moraes, M. O.; Lima, P. D. L.; Rabenhorst, S. H. B.; Leyva, A.; Burbano, R.R.; Teratog., Carcinog., Mutagen. 2003, 23, 215.

10. Ferreira, M. A. D.; Nunes, O. D. R. H.; Fujimura, A. H. Y.; Pessoa, O. D. L.; Lemos, T. L. G.; Viana, G. S. B.; Res. Commun. Mol. Pathol. Pharmacol. 1999, 106, 97.

11. Ferreira, M. A. D.; Nunes, O. D. R. H.; Leal, L. K. A. M.; Pessoa, O. D. L.; Lemos, T. L. G.; Viana, G. S. B.; Biol. Pharm. Bull. 2003, 26, 595.

12. Ferreira, M. A. D.; Nunes, O. D. R. H.; Fontenele, J. B.; Pessoa, O. D. L.; Lemos, T. L. G.; Viana, G. S. B.; Phytomedicine 2004, 11, 315.

13. Pessoa, O. D. L.; Lemos, T. L. G.; Rev. Bras. Farmácia 1997, 78, 9.

14. Mecca, S. B.; Proc. Sci. Sect. Toilt. Goods Assoc. 1963, 39, 7.

15. Costa, J. G. M.; Lemos, T. L. G.; Pessoa, O. D. L.; Monte, F. J. Q.; BrazFilho, R.; J. Nat. Prod. 1999, 62, 1044.

16. Costa, J. G. M.; Menezes, E. A.; Pessoa, O. D. L.; Lemos, T. L. G.; Flavour Fragr. J. 2004, 19, 461.

17. Menezes, J. E. S. A.; Lemos, T. L. G.; Silveira, E. R.; Braz-Filho, R.; Pessoa, O. D. L.; J. Braz. Chem. Soc. 2001,12, 787.

18. Hashidoko, Y.; Tahara. S.; Mizutani, J.; Phytochemistry 1994, 35, 325.

19. Evans, F. E.; Miller, D. W.; Cairns, T.; Baddeley, G. V.; Wenkert, E.; Phytochemistry 1982, 21, 937. 